\title{
STRUCTURAL INTERPRETATION OF THE MECHANISM OF POLYMER NETWORK FORMATION
}

\author{
T.E. Lipatova \\ Institute of Macromolecular Chemistry, Ukrainian Academy of Science, \\ Kiev, USSR
}

\begin{abstract}
Two types of network formation affected by physical factors are discussed: (1) the effect of strong intermolecular interactions in the formation of polyurethane networks, and (2) the effect of strong intermolecular repulsion operative during anionic polymerization of oligoester acrylates. The intermolecular interactions influence also the formation of supermolecular structures and the reaction kinetics.
\end{abstract}

During recent years much attention has been given to the possibility of the occurrence of some kind of local ordering in polymerizing systems. This ordering is considered to be a result of intermolecular interaction ${ }^{1-6}$. Many experimental structural and kinetic data can be explained if we take into account the existence of such regions ${ }^{7}$. These data allow us to think that for the most part the reactions of polymer molecule formation, especially in melt, are not those of isolated molecules. Many reactions, even in solution. are reactions between molecules which are found at an interface with some kind of supermolecular structures, composed perhaps of molecular aggregates $^{8}$.

According to theory ${ }^{9-12}$ and some experimental data, the reactions of three-dimensional polymerization or polycondensation are microheterogeneous $^{13-15}$. It seemed ${ }^{16}$ that at a definite stage of reaction in the reaction system there arise some particles of the colloid size (microgel). The chemical interaction between these three-dimensional particles leads to gel formation. Experimental data are in accordance with this mechanism ${ }^{17}$. There are several possible reasons for the arising of microheterogeneity in the oligomer and polymer systems. First, the strong intermolecular interaction which can lead to the formation of molecular aggregates even in the initial oligomer system before the beginning of the reaction. A typical example of such a system is that in which the reaction of polyurethane formation proceeds. Secondly, an incompatibility of components in the course of the reaction. This case is also typical of polyurethane systems. Thirdly, the difference in the reaction ability of functional groups which take part in three-dimensional polymerization and polycondensation ${ }^{18}$. 
In this way we can conclude that the principal cause of the microheterogeneity is the different level of intermolecular interaction between different blocks forming the polymer, if it is formed from oligomers or during the reaction.

Structural transformations in the system. especially the liquid-solid transition, influence essentially, we believe, the mechanism of polymer network formation. These transformations lead to a deviation from the theoretical predictions recently advanced.

The mechanism of gel formation is a key to the understanding of the mechanism of network polymer formation as well as the mechanism of the formation of supermolecular structures. Unfortunately, there are only a few papers on gel formation in the process of three-dimensional polymerization $^{17-19}$.

In the present article we shall discuss the mechanism of polymer network formation for two antipodal systems-polyurethanes which can be formed by the polycondensation (or polyaddition) reaction, and living network polyester acrylates obtained by anionic polymerization ${ }^{20-22}$.

The choice of these two systems was determined by the following factors. In both cases oligomer molecules take part in network formation. For polyurethane systems we used the oligoesterdiols or macrodiisocyanates ${ }^{23}$. There are strong intermolecular interactions in the polyurethane systems which continuously change in the course of the process. Investigating these particular systems, we aimed at clearing up the role of the intermolecular interactions in microheterogeneity arising during network formation. Oligoester acrylates were chosen to exemplify a system where between polymer chains strong forces of intermolecular repulsion are operative and the network is formed by branched chains with similar negative charges.

We have studied polyurethane formation from the initial stage to the end of hardening of the system beyond the gel point. In the course of the reaction we measured the viscosity changes, molecular weights and their distribution. heat capacities and some structural characteristics of the reaction products.

For the initial stage of the reaction (conversion 45-50 per cent), i.e. before a sharp increase in viscosity, it was found that network formation can be described as a reaction of the second order ${ }^{23}$. For this reaction we took

Table 1. Values of the reaction rate constants $k$ and activation energies $E$ for the initial stage of reaction of macrodiisocyanates (MDI, with different molecular weights) and trimethylolpropane

\begin{tabular}{ccccc}
\hline \multirow{2}{*}{$T,{ }^{\circ} \mathrm{C}$} & \multicolumn{4}{c}{$k \times 10^{2}, 1000$ g/equiv. NCO min for MDI using: } \\
\cline { 2 - 5 } & PPG-500 & PPG-1000 & PPG-2000 & PTMG-1000 \\
\hline 100 & 20.5 & 11.1 & 6.10 & 8.77 \\
80 & 6.40 & 4.40 & 2.40 & 5.55 \\
70 & 4.75 & 3.12 & 1.80 & 4.10 \\
60 & - & 2.41 & 1.10 & 2.90 \\
\hline $\begin{array}{c}\text { Activation } \\
\text { energy, }\end{array}$ & 7.10 & 8.30 & 7.30 & 6.80 \\
kcal/equiv. NCO & & & & \\
\hline
\end{tabular}


macrodiisocyanates (MDI) synthesized from 4, 4'-diphenylmethane-diisocyanate and oligoesterdiols of a different molecular weight and chemical nature-polyoxypropyleneglycol (PPG) and polytetramethyleneglycol (PTMG). We used trimethylolpropane as a trifunctional crosslinking agent. Experimental data are presented in Table 1, which shows that the reaction rate constant increases somewhat while the diol molecular weight in MDI decreases. This fact may be explained by the change in the specific solvation of reacting groups in polar reaction media ${ }^{24,25}$. This statement has been proved for the systems under investigation. The activation energy at the initial stages for all macrodiisocyanates is practically the same and is in good agreement with the activation energy of the spontaneous reaction of urethane formation in polar solvents ${ }^{24,25}$.

The calculation of the specific rate $w_{\mathrm{sp}}$ (or rate constant) for different conversion degrees $(\alpha)$ has shown that the reaction of polyurethane network formation obeys the usual kinetic laws (Figure 1; cf. reference 26).

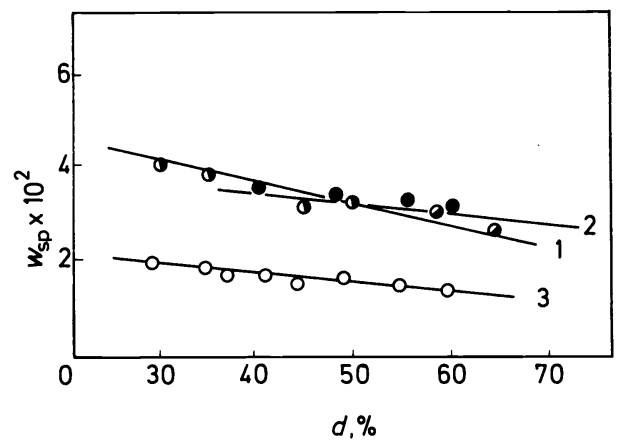

Figure 1. The dependence of the specific reaction rate $\left(w_{\mathrm{sp}}\right.$, equiv. $\left.\mathrm{NCO} / 1000 \mathrm{~g} \min \right)$ on the conversion degree for MDI based on PPG-5000 (1), PPG-1000(2) and PPG-2000 (3)

We have pointed out that in order to understand the mechanism of network formation it is important to have information about the structural changes in the system in the course of chemical reaction. For this purpose we have studied changes in the intrinsic viscosity of the reaction products. the molecular weights and their distribution characterized by the parameter $M_{z} / M_{w}$, and the mean-square inertia radius of the macromolecule, $\overline{R^{2}}$, in the course of reaction ${ }^{27,28}$. Some typical results are given in Table 2 and Figure 2(a). It can be seen that the sharp increase in intrinsic viscosity takes place at a conversion degree of 50-55 per cent. The molecular weights were determined by the method of progressive diffusion ${ }^{29}$ and the chain dimensions were calculated according to Tsvetkov and Klenin ${ }^{30} . M_{z}$ and $M_{w}$ were determined by the sedimentation method.

We can see from the experimental data that the molecular weights and polydispersity of the systems change very slightly up to 55 per cent conversion. This is in good agreement with the values of intrinsic viscosity. Beyond 55 per cent conversion a sharp increase in molecular weights (Table 2; 
T. E. LIPATOVA

Table 2. Dependence of $M, D .\left(\bar{R}^{2}\right)^{\frac{1}{2}}$, and $\widehat{K}^{2} / M$ on the degree of conversion for MDI based on PPG-1000

\begin{tabular}{cccccc}
\hline $\begin{array}{c}\text { Degree of } \\
\text { conversion } \\
\%\end{array}$ & $M$ & $\begin{array}{c}D \times 10^{-6} \\
\mathrm{~cm}^{2} / \mathrm{s}\end{array}$ & $\begin{array}{c}{[\eta] .} \\
\mathrm{dl} / \mathrm{g}\end{array}$ & $\begin{array}{c}\left(\overline{R^{2}}\right) \\
\AA\end{array}$ & $\overline{R^{2} / M}$ \\
\hline 0 & 1480 & 3.95 & 0.075 & 16.8 & 0.192 \\
15 & 1560 & 3.93 & 0.073 & 17.0 & 0.186 \\
25 & 1570 & 3.90 & 0.074 & 17.0 & 0.185 \\
30 & 1570 & 3.92 & 0.074 & 17.0 & 0.185 \\
51 & 1400 & 3.80 & 0.087 & 17.2 & 0.210 \\
63 & 7150 & 2.23 & 0.086 & 29.7 & 0.124 \\
66 & 19500 & 1.51 & 0.102 & 44.0 & 0.100 \\
69 & 57000 & 1.00 & 0.120 & 60.0 & 0.077 \\
\hline
\end{tabular}

(a)
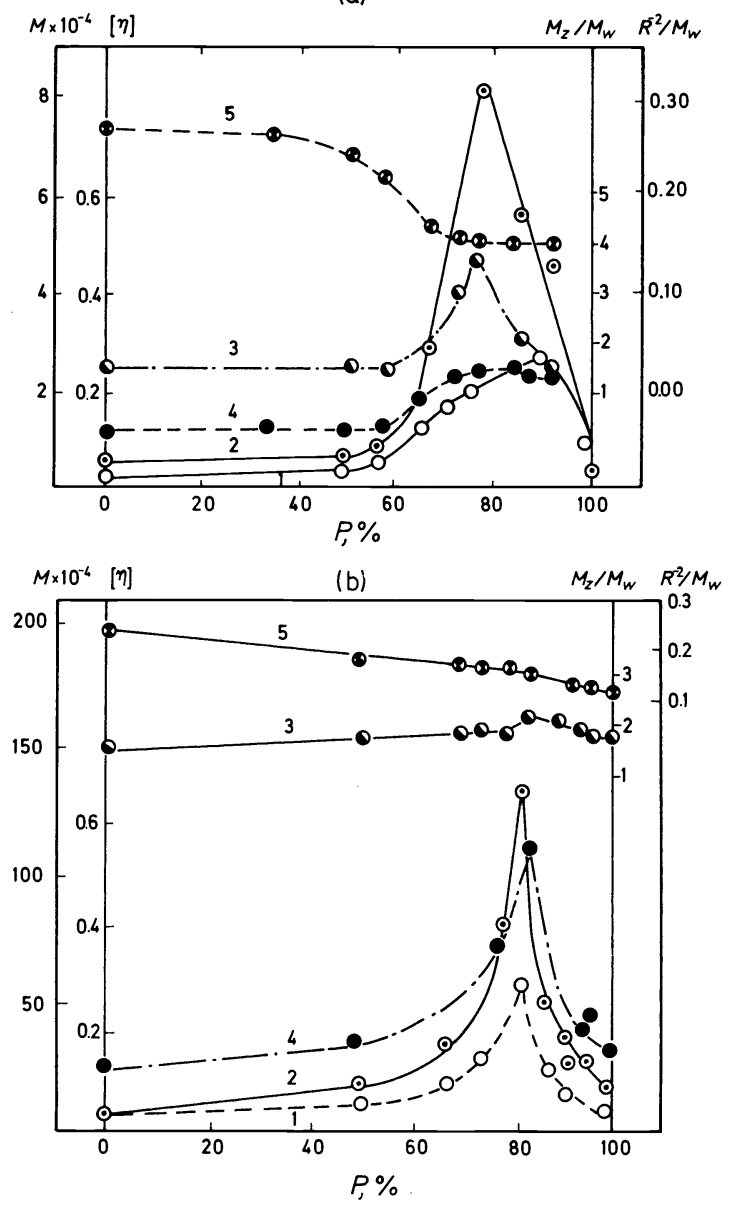

Figure 2. The dependence of molecular weights $M_{w}(1)$ and $M_{z}$ (2), the ratio $M_{z} / M_{w}(3)$, intrinsic viscosity $(\eta)(4)$ and ratio $\overline{R^{2}} / M(5)$ for the reaction products MDI (PPG-2000) and trimethylolpropane at $70 \mathrm{C}(\mathrm{a})$ and $125^{\circ} \mathrm{C}$ (b) on the degree of conversion 
Figure $2 a$ ), $M_{z}, M_{w}$ and their ratio. is observed. The increase in $M_{z} / M_{w}$ may be considered as a sharp broadening of the molecular weight distribution of the reaction products. In the region of this degree of conversion an intensive process of chain branching sets in. One can see this from the decrease in $\overline{R^{2}} / M$ with increase in molecular weight. In addition, $b$ in the equation $D=K M^{b}$ for reaction products obtained at this stage is 0.38 . this value being characteristic of highly branched polyfunctional chains ${ }^{29}$.

The macromolecular growth in the region of 60-86 per cent conversion proceeds with a similarity of macromolecular form being observed. as we can see from a slight increase in $[\eta]$ in the conversion region near the gel point and from the decrease in $\overline{R^{2}} / M$ (Table 2; Figure 2a). The broadening of the molecular weight distribution near the gel point is probably connected with the non-proportional growth of branched macromolecules due to the high viscosity of the system.

Thus we can conclude that in systems with strong intermolecular interactions, in which some molecular aggregates may be present, the reaction rate is described by ordinary kinetic equations. The molecular aggregates are not yet the stable elements of rearrangement and have no interface.

The investigation of the sol fraction beyond the gel point showed that the polydispersity of soluble reaction products diminishes (Figure 2a). Thus we may suppose that in gel formation large branched molecules take part first. However, beyond the gel point, $M_{w}$ of the sol fraction increases in the course of the reaction, whereas $[\eta]$ and $\overline{R^{2}} / M$ remain constant in the wide range of degree of conversion. This can be related with such a growth of macromolecules when the particles preserve their shape. If we take into account that at 85 per cent conversion (beyond the gel point. calculated according to Flory) the weight fraction of sol is $\sim 58$ per cent and the molecular weight of sol increases, it becomes evident that a continuous network is formed only at the last stages of conversion.

If, as we believe, intermolecular interactions play such an important role in the formation of network polymers, it should be very useful to study the influence of the temperature and route of reaction (in one or two stages) on that stage of reaction which precedes gel formation.

From a comparison of the data given in Figure 2(a) and Figure $2(b)$ it is obvious that at 50 per cent conversion $M_{v},[\eta]$ and polydispersity of the reaction products at $125^{\circ} \mathrm{C}$ are somewhat higher than at $70^{\circ} \mathrm{C}$, whereas $\overline{R^{2}} / M$ is lower. This testifies to the fact that more branched macromolecules with a higher molecular weight are formed at $125^{\circ} \mathrm{C}$. Beginning from 50 per cent conversion at $125^{\circ} \mathrm{C}$, a very sharp increase in $M_{z}$ and $M_{n}$ takes place in comparison with the same values at $70^{\circ} \mathrm{C}$. However, the changes in polydispersity of polymer molecules formed at $125^{\circ} \mathrm{C}$ are less pronounced than at $70^{\circ} \mathrm{C}$.

The dependence of $\log [\eta]$ and $\log D$ on $\log M$ for polymers obtained at $125^{\circ} \mathrm{C}$ is usual for linear polymer homologues in good solvents, in contrast to the situation with polymers formed at $70^{\circ} \mathrm{C}$. Thus at $125^{\circ} \mathrm{C}$ and 50 per cent conversion a chiefly linear uniform growth of the polymer chains takes place. At the gelation the polydispersity increases (at 82 per cent conversion the ratio $M_{z} / M_{w}$ reaches 2.2). Beyond the gel point the process at $125^{\circ} \mathrm{C}$ differs from that at $70^{\circ} \mathrm{C}$. 
The polydispersity of the sol fraction at $125^{\circ} \mathrm{C}$ remains practically invariable, whereas $M_{z}, M_{w}$ and [ $\left.\eta\right]$ decrease in the course of gel formation. This can be explained by more complex molecules being involved in gel formation from the sol fraction.

The observed differences in the process of network formation which proceeds at different temperatures may be explained by a diminishing in the intermolecular interaction in the system at $125^{\circ} \mathrm{C}$. This leads to the predominant growth of linear chains in distinct regions associated with degrees of conversion.

The changes in the intermolecular interaction are confirmed by the i.r. spectra of MDI. The increase in temperature up to $125^{\circ} \mathrm{C}$ leads to a decrease in the intensity of the absorption band at $3340 \mathrm{~cm}^{-1}$. This is the band connected with the NH-groups participating in the H-bond formation. At the same time a band at $3486 \mathrm{~cm}^{-1}$ arises in the spectra, which can be attributed to the free $\mathrm{NH}$-groups ${ }^{31,32}$. Thus the increase in temperature leads to the same change in the mechanism of polyurethane network formation.

Up to now we have discussed only the mechanism of the reaction which proceeds in two stages. It was also necessary to study this process under conditions in which all components (diisocyanate, diol and triol) are mixed together at the same time. In principle, the character of the kinetic curves here does not differ from those for a one-stage process ${ }^{23}$.

To understand the mechanism of network formation it is important to study the reaction beyond the gel point ${ }^{33}$. We studied the kinetics of network formation by the calorimetric method. The thermogram of the process can be divided into two parts: first, to the gel point (according to Flory), i.e. to $\sim 70$ per cent conversion, and, second, beyond the gel point. The last part can be considered as an independent curve, taking into account that at the gel point the gel is absent ${ }^{34}$. In this case the heat effect is equivalent to the

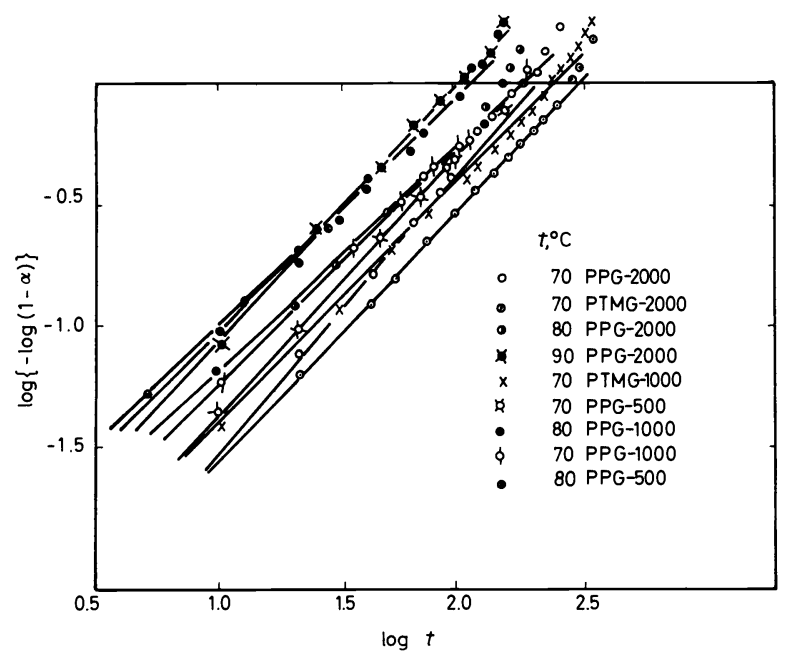

Figure 3. The dependence $\log \{-\log (1-\alpha)\}$ on time for polyurethane network formation beyond the gel point at $70 \mathrm{C}$ and $90^{\circ} \mathrm{C}$ 
fraction of the substance transformed into gel. Some of our results are presented in Figure 3 in the coordinates of the Avrami equation for phase transition ${ }^{35}$. It is seen that the process beyond the gel point can be described by this equation. The one-stage process also can be described in the same manner. The parameters of the equation, $K$ and $n$, were calculated for the systems under investigation (Table 3 ). It is seen from Table 3 that the gelation

Table 3. Values of the parameters $K$ and $n$ of the Avrami equation for polyurethane systems

\begin{tabular}{llll}
\hline System based on & $\begin{array}{c}\text { Reaction } \\
\text { temperature, }{ }^{\circ} \mathrm{C}\end{array}$ & $n$ & $K \times 10^{3}, \mathrm{~min}^{-1}$ \\
\hline
\end{tabular}

\begin{tabular}{lllc} 
Two-stage method & & \\
PPG-2000 & 70 & 1.0 & 5.79 \\
PPG-1000 & 70 & 1.05 & 11.0 \\
PPG-500 & 70 & 1.0 & 13.6 \\
PPG-2000 & 30 & 1.13 & 7.29 \\
PPG-1000 & 80 & 1.0 & 15.9 \\
PPG-500 & 80 & 1.0 & 23.0 \\
PPG-2000 & 90 & 1.01 & 15.9 \\
PTMG-2000 & 70 & 1.0 & 5.4 \\
PTMG-1000 & 70 & 1.05 & 8.0 \\
\multicolumn{1}{c}{ One-stage method } & & \\
PPG-2000 & 80 & 1.0 & 6.36 \\
PPG-1000 & 80 & 1.0 & 11.3 \\
PPG-500 & 80 & 1.0 & 13.0 \\
\hline
\end{tabular}

process is described in practice by a first-order equation $(n \sim 1)$. The change in the reaction order and at the same time the liquid-solid transition and interface formation (formation of microheterogeneity) show that the chemical process is governed by the rate of structure formation in the reaction system, i.e. by the total surface of the microgel particles. For this reason the use of the Avrami equation for describing the reaction kinetics in this stage may be more correct than the use of the kinetic equation of the first order. To confirm our point of view, we have studied the reaction system by different physical methods ${ }^{36,37}$. Figure 4 shows the change in the heat capacity of the system based on PPG-2000 in the course of glass-liquid transition. These and other results show the dependence of the glass transition temperature, $T_{g}$, and interval (the difference between temperatures at the outset and the end of the transition) on the degree of conversion (Figure 5). Here $T_{\mathrm{g}}$ was determined as a temperature at which the value of the leap in the heat capacity reaches one-half of the full value.

It is seen from Figure 5 that the glass transition temperature and interval do not change up to 40 per cent conversion and after this the values sharply change in the interval from 40 to 70 per cent.

Figure $4(b)$ shows the dependence of $C_{p}$ on temperature in the region of the liquid-solid transition for the reaction mixture at 50-55 per cent conversion. We must note that there are inflection points on the curves in the transition regions. The same character is exhibited by the curve of the dependence of $C_{p}$ on temperature in the glass transition region for the system 
(a)

$c_{p} \cdot \frac{\mathrm{cal}}{\mathrm{g} \cdot \mathrm{grad}}$
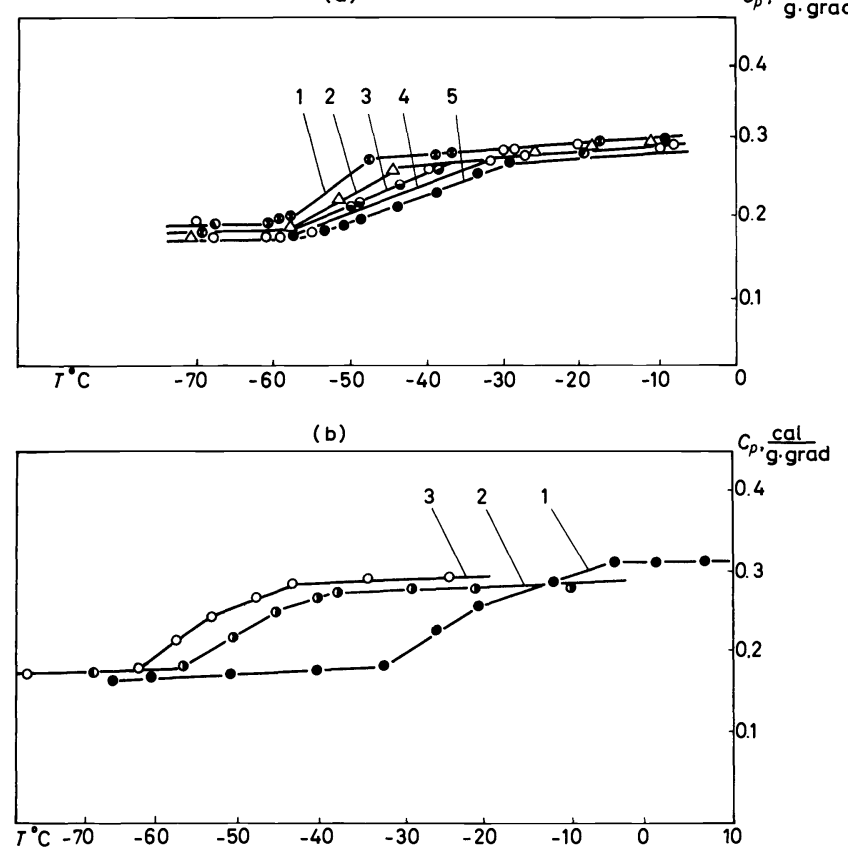

Figure 4. The temperature dependence of $C_{p}$ for the reaction system based on (a) PPG-2000 at degrees of conversion $35 \%$ (1), $55 \%$ (2), $75 \%$ (3), $85 \%$ (4), $100 \%$ (5), and based on (b) PPG-1000

(1) and PPG-2000 $(2,3)$ at $50 \%(1,2)$ and $10 \%$ (3). In case 3 of (b) $10 \%$ aerosil was added

MDI based on PPG-2000-trimethylolpropane even at ten per cent conversion but in the presence of five per cent filler (aerosil) (Figure 4b, curve 3). We can see that all curves which were determined have a break at a definite degree of conversion, being constant before and beyond this conversion region. The latest sharp break takes place at $85-98$ per cent.

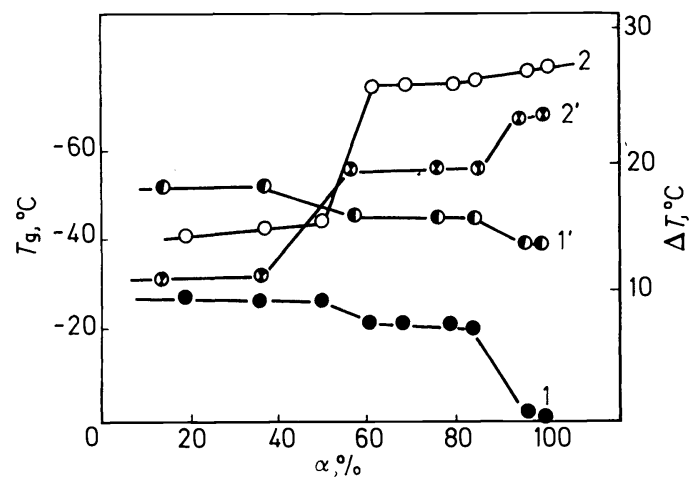

Figure 5. The dependence of the glass transition temperature $\left(1,1^{\prime}\right)$ and glass transition temperature interval $\left(2,2^{\prime}\right)$ on the degree of conversion for systems based on PPG-1000 $\left(1,2^{\prime}\right)$ and PPG-2000 (1', 2) 
The broadening of the glass transition interval (Figure 5) may be connected with the irregularity of the free volume distribution ${ }^{38,39}$ due to increase of local ordering in the polymer system ${ }^{38}$. Indeed, in our case microgel particles can appear as a result of the chemical crosslinking in microregions, surrounded by macromolecules of a smaller degree of complexity.

The relaxation processes in microregions would have different rates. It is possible that this is the reason for some breaks in the heat capacity curves. This view is favoured by the results obtained for the system with low conversion ( 10 per cent) in presence of the filler (Figure $4 b$, curve 3 ). The aerosil particle can adsorb MDI molecules on its surface and even interact with them chemically. Such particles may be considered as analogous to the microgel particles. Then the relaxation properties in this system also must be analogous to those of the unfilled system which contains microgel particles (in our case it is the system at 55 per cent conversion). We have in fact observed ${ }^{36}$ this analogy (Figure $4 b$ ). Therefore it can be concluded that in the region of 40-70 per cent conversion microgel particles are formed which are the centres of network formation; at the same time microdividing into two phases proceeds.

The stability of the glass transition parameters at $75-85$ per cent conversion shows that in this region the system does not undergo any essential structure changes. The upward shift of $T_{\mathrm{g}}$ at a conversion above 85 per cent is probably connected with a large decrease in the molecular mobility of the chains due to monolithic network formation. These suppositions, although logical, have still to be proved. For this purpose we have studied formation by the method of turbidity spectra, whicn allows us to observe the appearance of the microgel particles, if any, their size and concentration as well as their behaviour in the course of network formation.

One- and two-stage reactions were investigated ${ }^{37}$. Some results for twostage synthesis are given in Figure 6. It is seen that at 70 per cent conversion particles of colloid size $\left(r_{w}=680 \AA\right)$ are formed in the system. Investigation ${ }^{37}$ leads us to conclude that these particles are formed by crosslinked macromolecules and are not aggregates of the latter. Beginning from the moment

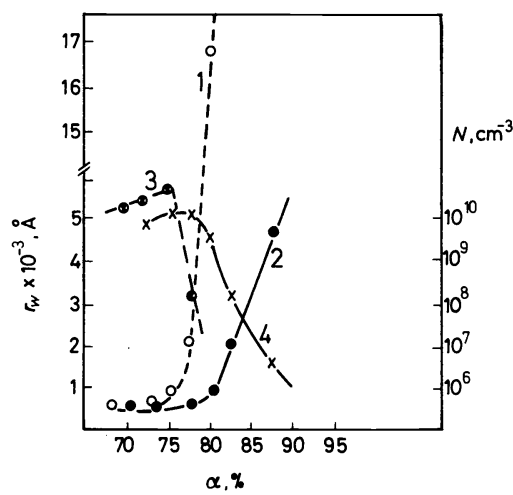

Figure 6. The dependence of the size $r_{w}(1,2)$ and particle number $N(3.4)$ on the degree of conversion for the reaction products based on PPG-1000 (1, 3), PPG-2000 $(2,4)$. Concentration of solution $5.05 \%$ 
of their appearance, the number of particles in the system increases, whereas their size does not change. After reaching a definite concentration, there is a sharp increase in the size of the particles and a decrease in their number. Thus we may suppose that the increase in the size of the particles is connected with direct interaction between them or with their bonding by molecules of great complexity. The increase in the number and size of the particles is more pronounced in the system based on PPG-1000 than in that based on PPG-2000 (Figure 6).

The one-stage reaction may be characterized by the same curves but the network microparticles arise there at the earlier stages of the reaction (55 per cent conversion). Beyond this conversion there is a region where the size and number of the particles do not change, and their concentration sharply increases. At 72.5 per cent conversion (for PPG-1000) and 75 per cent (PPG-2000) the reaction mixture becomes insoluble and can only swell, being in contact with the solvent.

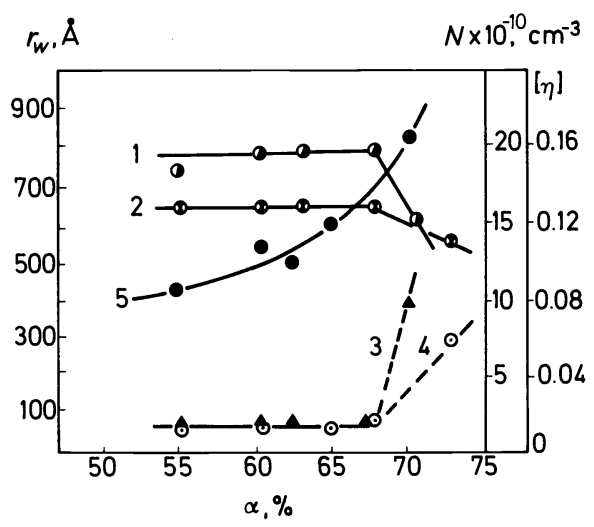

Figure 7. The dependence of the size $r_{w}(3,4)$, particle number $N(1,2)$ and intrinsic viscosity (5) on the degree of conversion for systems based on PPG-1000 $(1,3,5)$ and PPG-2000 $(2,4)$ for the one-stage reaction. Concentration of solution $5.05 \%$

It can be seen that, in contrast to theoretical suppositions, the gelation process is shifted to the region of lower degrees of conversion in the one-stage process in comparison with the two-stage one. In both methods of synthesis some deviations are found from the position of the gel point calculated theoretically. These deviations cannot be explained only by the differences between the conditions of microsegregation for the different methods of synthesis. Indeed, in the polyurethane systems there exist strong intermolecular interactions ${ }^{40}$ which may lead under definite conditions to the microdividing of the system into two phases. For the one-stage process, when the conversion degree is not high ( 40 per cent), not only polyurethane molecules but also molecules of unreacted diisocyanate, esterglycol and triol are present in the system, which is thus more inhomogeneous than for the two-stage reaction. Because of this the microdividing in such a system begins at lower degrees of conversion. The addition of triol and diisocyanate molecules to the molecular aggregates leads to the formation 
of microgel particles (network microparticles) (Figure 7). Diisocyanate and triol being exhausted, the formation of new microparticles becomes less probable as the molecular complexity at this stage is not high. Further in the course of the reaction the molecular weight of the sol fraction increases (increase in $[\eta]$; Figure 7 ) and at 70 per cent conversion the molecular complexity becomes high. This can lead to the formation of new particles, and thus (taking account of particles already formed) their concentration very quickly reaches the critical value at a particular degree of conversion, which is lower than that for the two-stage process.

All the results discussed above allow us to conclude that independently of the method of synthesis two periods of gel formation can be distinguished:(1) the formation of network particles of the colloid size in coexistence with the sol fraction and (2) the formation of a monolithic network (structural gelation) due to the bonding of the particles at their critical concentration. Thus we have an interval, not a point, of gelation for systems where strong intermolecular interactions are operative and influence essentially the mechanism of network formation. In such systems the reaction probably proceeds in three different regions with different rate constants in microgel particles, on their surface and in the space between the microgel particles.

The experimental data concerning the structure ${ }^{41}$ and relaxation behaviour $^{42,43}$ of network polyurethanes are in good agreement with the mechanism of network formation given here ${ }^{23}$. The amorphous network polyurethanes have a globular structure ${ }^{41}$ and their relaxation properties are dependent on the globule size ${ }^{43}$, which is connected in its turn with the molecular weight of the oligomer molecule.

Now we shall consider a system where the formation of network structure proceeds in the course of the anionic polymerization of oligoesteracrylates in tetrahydrofurane in presence of sodium naphthalenate. In this case the network is formed from large branched chain fragments possessing from one to three charges per oligomer block. The main features of the anionic polymerization were formulated by us earlier for $\alpha, \omega$-methacryl-bis-triethyleneglycolphthalate:

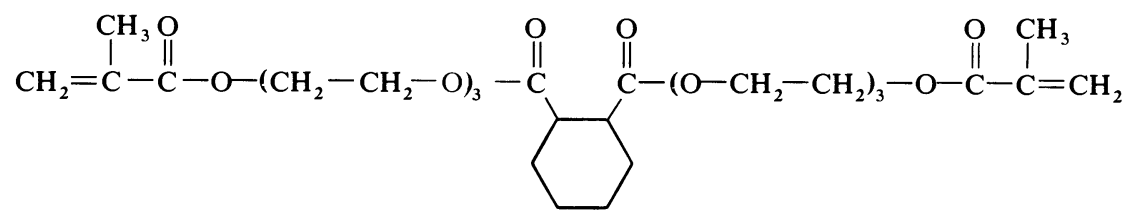

One of the distinguishing features in the initiation of the anionic polymerization of oligoesteracrylates is the existence of the so-called 'limit' concentrations of the catalyst below which the polymerization cannot practically take place ${ }^{44}$. In the initiation, equilibrium in the electron distribution between the functional groups with different electron affinities ${ }^{20}$ is quickly established. The existence of the 'limit' concentration of the catalyst is due to the fact that the phthalate groups possess a higher electron affinity than the methylmethacrylate groups and the equilibrium here is shifted to the charged phthalate groups. Only at a definite catalyst concentration do 
we obtain such a concentration of electron-possessing methylmethacrylate groups as that at which the initiation of the polymerization proceeds.

In the course of chain and network formation a free oligomer-soluble polymer-network polymer equilibrium is usually established ${ }^{21,44}$. In these conditions living network polymers are formed ${ }^{22}$.

It is well known that the formation of a soluble branched polymer precedes gelation. Therefore we think it desirable to discuss some laws which govern the formation of the soluble polymer and trace the connection between the

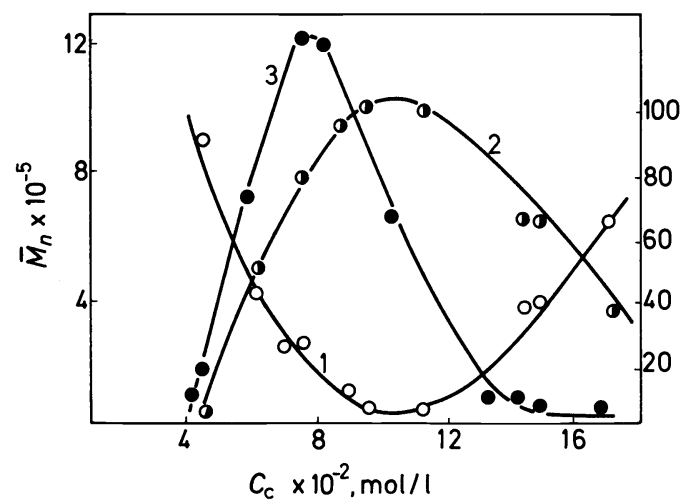

Figure 8. The dependence of the yield of network (2) and soluble (1) polymers and molecular weight $M_{n}(3)$ on the catalyst concentration $\left(C_{\mathrm{c}}\right)$

soluble and the network polymer. In our work we have established that the soluble polymer yield increases with the catalyst concentration, whereas the network polymer yield passes through a maximum at a catalyst concentration $\sim 0.1 \mathrm{~mol} / \mathrm{l}$ at $25^{\circ} \mathrm{C}$ (cf. ref. 22 ).

Figure 8 shows the dependence of the yield of soluble (curve 1) and network (curve 2) polymer and the molecular weight of soluble polymer (curve 3) on the catalyst concentration. Here the total yield is accepted to be 100 per cent. It is seen that at a catalyst concentration of $0.1 \mathrm{~mol} / \mathrm{l}$ the yield of the network polymer has its maximum and the soluble polymer yield has its minimum. The molecular weight of soluble polymer also passes through a maximum $\left(10^{6}\right)$ at $\sim 0.07 \mathrm{~mol} / 1$. This molecular weight cannot be higher and characterizes the size of that chain fragment which takes part in network formation. For the polymer with a molecular weight of $0.35 \times 10^{5}$ the constant $a$ in the Mark-Houwink equation is 0.39 , which is typical of a branched polymer.

Thus we can conclude that the network polymer formed by the anionic mechanism from oligoester acrylates consists of branched rigid fragments. The greater the molecular weight of the polymer the higher the degree of branching. The rigidity of chains bearing the negative charges may be seen from the values of the relative viscosity of the same solutions of the living and dead polymers which are given in Table 4.

Thus in this case network formation proceeds without the stage of microgel. In the equilibrium state the system in which the reaction proceeds may be 
characterized by the equilibrium contents of living soluble polymer and network polymer and free oligomer. Here the molecular weight distribution in the living soluble polymer was found to be narrow. Therefore the network is formed by fragments of the same size. It was very interesting to find out in which way the mechanism of network formation influences the structure of the amorphous network polymer. From the two electron micrographs in Figure 9 it is seen that 'radical' and 'anionic' network polymers obtained from the same oligomer ( $\alpha, \omega$-methacryl-bis-triethyleneglycolphthalate) have different structures. The supermolecular structure formation in the 'radical' network polymer was described by $\operatorname{Berlin}^{45}$ (Figure $9 a$ ). We should

Table 4. Relative viscosity of living and deactivated soluble polymer obtained at different catalyst concentrations $\left(C_{\mathrm{c}} \mathrm{mol} / \mathrm{l}\right)$ at $25^{\circ} \mathrm{C}$ in solution, polymer concentration in solution $0.6 \mathrm{~mol} / 1$

\begin{tabular}{lcc}
\hline \multicolumn{1}{c}{$C_{\mathrm{c}}$} & $\eta_{\text {rel }}$ (living polymer) & $\eta_{\text {rel }}$ (deactivated polymer) \\
\hline 0.019 & 2.15 & 1.85 \\
0.024 & 2.43 & 2.21 \\
0.028 & 2.70 & 2.56 \\
0.06 & 2.96 & 2.64 \\
\hline
\end{tabular}

therefore like to discuss only the structure formation for living network polymers. When a living network polymer is formed, the microgel step is absent. The equilibrium living network polymer present in the reaction system may be described as a loose swollen gel. After deactivation and vacuum drying, the polymer unsaturation remains low, being slightly dependent on catalyst concentration. The value of $M_{c}$ (molecular weight between crosslinks) also changes only very little. Thus we can conclude that after the charge has disappeared, the usual intermolecular bonds between chains are recovered; at the same time a further process of crosslinking goes on with relaxation, and supermolecular structures are formed. As a result of these different processes the network structure is as presented in Figure $9(b)$. It is possible that this type of structure consists of high-density domains, as has been supposed ${ }^{46}$ for linear amorphous polymers. These structures have a higher level of organization than the globular ones and the properties of the polymer will be different from those of the globular polymer of the same chemical nature ${ }^{47}$.

Thus a specific feature in network formation from oligomer molecules by any mechanism is the strong intermolecular interaction between the molecules, which influences the chain conformation and supermolecular structure.

In conclusion it is worth noting one of the most interesting features of the anionic polymerization of oligomers, viz. the equilibrium between living network and soluble polymer and free oligomer in the system under consideration. At each equilibrium reaction stage both the polymerization and depolymerization proceed at the same time. It is well known that we can reach the equilibrium from two directions : by direct reaction (polymerization) and by the reverse reaction of depolymerization. Therefore a question arises 


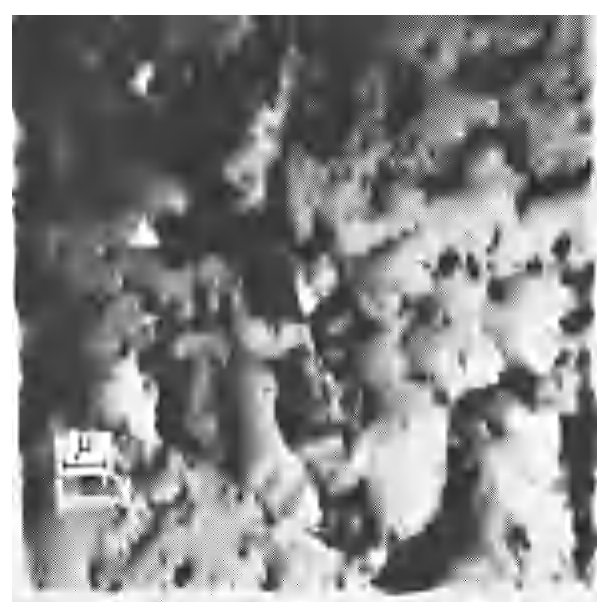

(a)

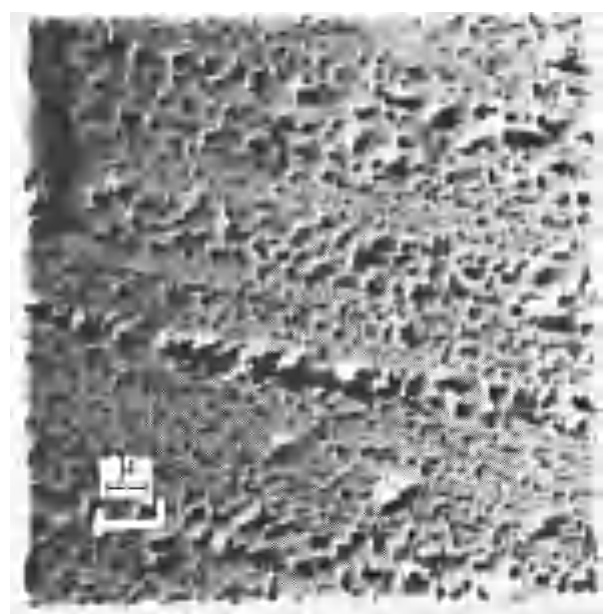

(b)

Figure 9. Electron microphotographs of the network polyesteracrylates obtained (a) by radical mechanism and (b) through living polymers

whether we can make 'living' the deactivated polymer via its contact with catalyst under conditions usual for the formation of living polymers.

We have discovered that under such conditions the system can reach its equilibrium state as a result of the network depolymerization in the presence of the catalyst. Here the equilibrium quantities of living network polymer and soluble living polymer and free oligomer are generated ${ }^{21}$. The scheme 
of the equilibrium may be presented as follows:

(oligomer) + (catalyst) -- initial system
$\downarrow$
(living network) + (living soluble polymer) + (oligomer) + (catalyst) - an
equilibrium system
$\uparrow$
(inactive network) + (catalyst) ----- initial system

Thus we have established the possibility of activation of the inactive polymer independently of the mechanism of its formation. The only condition for the activation is the ability of the monomer or oligomer to form living polymers by the anionic mechanism.

\section{REFERENCES}

1 A. A. Berlin, N. N. Tvorogov and G. V. Korolev, Izvest. Akad. Nauk. Ser. Chim. 193 (1966).

2 A. A. Berlin, N. N. Tvorogov and G. V. Korolev, Dokl. Akad. Nauk, 170, 1073 (1966).

3 G. V. Korolev and N. P. Tvorogov. Vysokomol. Soed. 6, 1006 (1964).

${ }^{4}$ G. Kalashnikova, M. V. Kazdan and Z. Y. Berestneva, Dokl. Akad. Nauk, 158, 939 (1964).

5 M. V. Kazdan, T. N. Dumaeva and V. A. Kargin, Vysokomol. Soed. 8, 204 (1966).

6 V. A. Kargin. I. V. Pismenko and E. P. Cherneva, Vysokomol. Soed. A10, 846 (1968).

7 T. E. Lipatova, V. M. Siderko and V. A. Budnikova, Vysokomol. Soed. 6, 910 (1964).

8 N. A. Platé, in Kinetics and Mechanism of Formation and Transformation of Macromolecules. Nauka: Moscow (1968).

9 K. Dušek, J. Polymer Sci. B3, 209 (1965).

10 K. Dušek, J. Polymer Sci. C16, 1289 (1967).

11 J. Seidl, J. Malinský, K. Dušek and W Heitz. Advanc. Polymer Sci. 5, 113 (1967).

12 K. Dušek, Polymer Preprints, 11, 536 (1970).

13 G. V. Korolev and A. A. Berlin, Vysokomol. Soed. 4, 1954 (1962).

14 G. V. Korolev and A. A. Berlin, Vysokomol. Soed. 4, 1520 (1962).

15 W. Funke, Advanc. Polymer Sci. 4, 157 (1965).

16 E. Bobalek. E. Moore, S. Levy and W. Heitz. Advanc. Polymer Sci. 8, 625 (1964).

${ }^{17}$ L. Gallacher and F. Batterheim, J. Polymer Sci. 58, 697 (1962).

18 W. Funke, Chimia, 22, 11 (1968).

19 K. Andrianov and V. Emeljanov, Vysokomol. Soed. A13, 1230 (1971).

20 T. Lipatova, G. Shapoval, E. Shevchuk and N. Basilevskaya, J.Macromol. Sci.: Chem. A5(2), 345 (1971).

21 T. Lipatova. G. Shapoval, N. Basilevskaya, E. Shevchuk and T. Novikova, Dokl. Akad. Nauk, 208, 645 (1973).

22 T. E. Lipatova, G. Shapoval, N. Basilevskaya and E. Shevchuk, J. Polymer Sci. C, N42, 1 (1973).

23 T. E. Lipatova, Vysokomol. Soed. A15, 327 (1973).

24 I. Pronina, Yu. Spirin, A. Blagonravova and A. Gantmacher, Kinetika i Kataliz, 7, 439 (1966).

25 A. Oberth and R. Brunner, J. Phys. Chem. 72, 845 (1968).

26 T. Lipatova and V. Ivaschenko, Vysokomol. Soed. A11, 2217 (1969).

27 A. Nesterov, T. Lipatova and V. Ivaschenko, Vysokomol. Soed. B12, 150 (1970).

28 T. Lipatova, A. Nesterov and V. Ivaschenko, Vysokomol. Soed. A12, 1039 (1970).

29 V. Tsvetkov, V. Eskin and S. Frenkel, Struktura Makromolekul v Rastvore. Nauka: Moscow (1964).

30 V. Tsvetkov and S. Klenin, J. Tech. Phys. 29, 1393 (1959).

31 T. Tanaka, T. Jokoima and K. Kaku, Mem. Fac. Engng Kyoto Univ. 23, 115 (1963).

32 Y. Boarchuk, L. Rappoport, V. Nikitin and N. Apuchtina, Vysokomol. Soed. 7, 778 (1965).

${ }^{33}$ T. Lipatova and S. Zubko, Dokl. Akad. Nauk, 184, 887 (1969).

34 P. Flory, Principles of Polymer Chemistry. Cornell University Press: Ithaca (1953).

${ }^{35}$ M. Avrami, J. Chem. Phys. 8, 212 (1940); 9, 177 (1941).

36 T. Lipatova and S. Zubko, Vysokomol. Soed. A12, 1555 (1970). 
37 A. Nesterov, T. Lipatova and S. Zubko, Vysokomol. Soed. A12, 2252 (1970).

38 P. Mason, J. Chem. Phys. 35, 1523 (1961).

39 P. Mason, Polymer, 5, 625 (1964).

40 Yu. Lipatov, Yu. Kercha and L. Sergeeva, Struktura i Svojistva Poliuretanov. Naukova Dumka: Kiev (1970).

41 T. Lipatova, V. Ivaschenko and L. Bezruk, Vysokomol. Soed. A13, 1701 (1971).

42 V. Pavlov, Yu. Lipatov, T. Lipatova, S. Zubko and G. Svjatnenko, Vysokomol. Soed. A11, 2784 (1969).

43 V. Pavlov, T. Lipatova and S. Zubko, Vysokomol. Soed. A13, 682 (1971).

44 T. Lipatova, G. Shapoval, N. Basylevskaya and E. Shevchuk, Vysokomol. Soed. A11, 2280 (1969).

45 A. A. Berlin, N. N. Tvorogov, G. V. Korolev, Dokl. Akad. Nauk, 170, 1073 (1966).

46 S. Aharon, J. Macromol. Sci. B7, 73 (1973).

47 T. Lipatova and V. Siderko, Dokl. Akad. Nauk, 178, 856 (1968). 